\title{
Growth Dynamics of Ga Nanodroplets on 2D Substrate
}

\author{
Zhaslan Baraissov ${ }^{1,2}$, Federico Panciera ${ }^{2,3}$, Jean-Christophe Harmand ${ }^{3}$, Gilles Patriarche ${ }^{3}$, Utkur \\ Mirsaidov ${ }^{1,2,4}$ \\ 1. Center for Advanced 2D Materials and Department of Physics, National University of Singapore, \\ Singapore. \\ 2. Center for BioImaging Sciences and Department of Biological Sciences, National University of \\ Singapore, Singapore. \\ 3. Centre for Nanoscience and Nanotechnology, CNRS, University of Paris-Saclay Marcoussis, France. \\ 4. Department of Materials Science and Engineering, National University of Singapore, Singapore.
}

Metal nanodroplets can be employed for many applications, such as the growth of nanowires [1], carbon nanotubes [2] and catalysis [3]. However, these processes require precise control of the size and distribution of the nanodroplets. For example, in the case of self-catalyzed nanowire growth, the droplet shape and size are crucial for the dimensions and crystal phase of the nanowire. Despite reported simulations [4-5] of the phenomenon, it is not fully understood how the nucleation and growth of the nanodroplets happen at the nanoscale.

Here we examine the growth dynamics of Ga nanodroplet on SiNx membrane using an environmental transmission electron microscope (ETEM) equipped with custom-made Molecular Beam Epitaxy (MBE) sources. Unlike the case of chemical vapor deposition, MBE does not need a step to decompose complex chemical precursors and deposition rate is constant in a large range of temperature. A first set of experiments was carried out by ramping up the substrate temperature in order to find the threshold temperature for significant $\mathrm{Ga}$ desorption. Then, we conducted isothermal experiments below this threshold temperature and we observe Ga droplet growth by coalescence and Ostwald ripening. In situ experiments demonstrate that seeds appear on specific sites of the membrane. The seeds later act as sinks where material is transported from surrounding areas through surface diffusion. We implemented an image processing algorithm that allows us to segment, track and measure more than hundred individual droplets in parallel. Based on the analysis, we show that the growth dynamics of the droplet follows a power law:

$$
R(t)=A\left(t-t_{0}\right)^{B}
$$

Where $t_{0}$ is the droplet nucleation time, $\mathrm{A}$ is a variable dependent on the deposition rate and on the surface diffusion coefficient, and B is the growth exponent. By examining these variables for over 300 nanodroplets and key growth parameters such as density of particles and distribution of sizes depend on flux and temperature, we show that heterogeneous nucleation and growth of the nanodroplets is driven by 2D surface diffusion of Ga clusters. These findings reveal how the flux of material and the surface diffusion affect the growth mechanisms.

\section{References:}

[1] Jacobsson, Daniel et al, Nature 531.7594 (2016), p. 317.

[2] Yudasaka, Masako et al, Applied Physics Letters 67.17 (1995), p. 2477-2479.

[3] Taccardi, N. et al, Nature chemistry 9.9 (2017), p. 862.

[4] Steyer, A. et al, Physical Review A 44.12 (1991), p. 8271. 
[5] Fritter, Daniela, Charles M. Knobler, and Daniel A. Beysens, Physical Review A 43.6 (1991), p. 2858

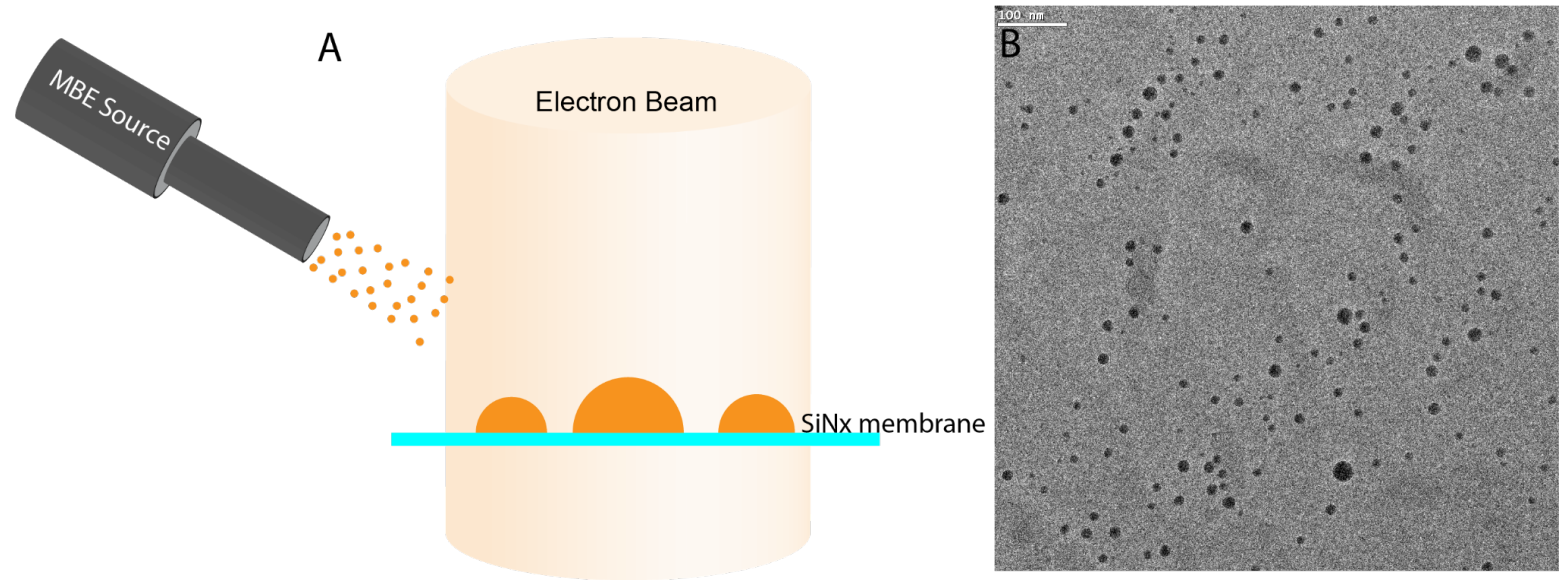

Figure 1. (A) Schematic of the in situ MBE deposition system at $\mathrm{C} 2 \mathrm{~N}$, using which we perform two sets of experiments a) constant source temperature at $940{ }^{\circ} \mathrm{C}$ and varying substrate temperature from $200{ }^{\circ} \mathrm{C}$ to $500{ }^{\circ} \mathrm{C}$; b) constant substrate temperature at $350{ }^{\circ} \mathrm{C}$ and different source temperatures in range from $910{ }^{\circ} \mathrm{C}$ to $940^{\circ} \mathrm{C}$. (B) Full field of view for substrate temperature of $250{ }^{\circ} \mathrm{C}$.

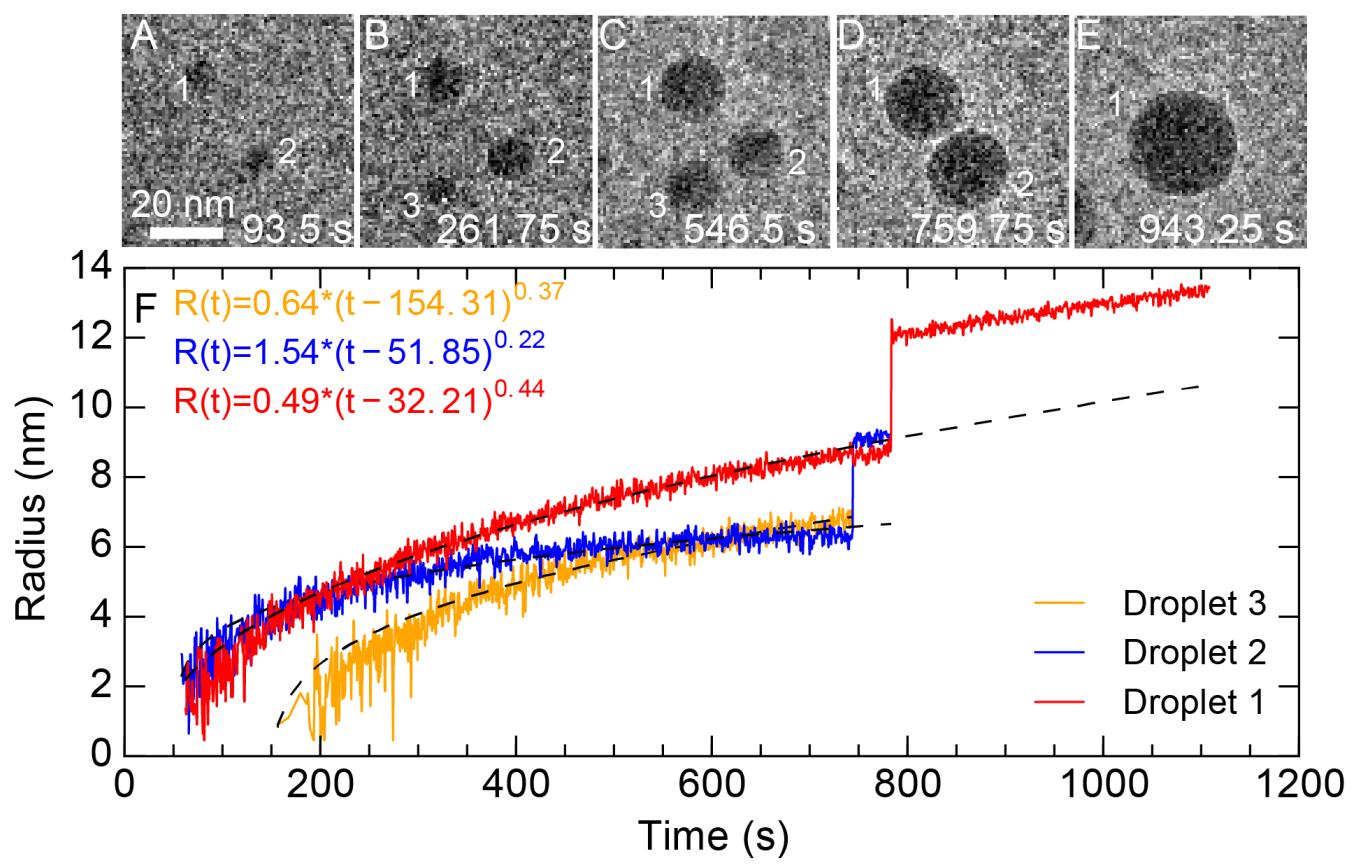

Figure 2. Growth and coalescence events for substrate at $300{ }^{\circ} \mathrm{C}$ and Ga source at $940{ }^{\circ} \mathrm{C}$ (A) Droplet 1 and 2 nucleate and grow. (B) Droplet 3 nucleates and grows. (C) Droplet 3 grows quickly and reaches same size as droplet 2. (D) First coalescence event, droplet 2 consumes droplet 3. (E) Second coalescence event, droplet 2 hops and merges with droplet 1. (F) Plot of droplet radius versus time for the three droplets. Here, we fit the growth curves to a power law with exponents $B_{1}=0.44, B_{2}=$ $0.22, B_{3}=0.37$. 